\title{
EXCHANGE CROSS SECTIONS FOR HARD BINARIES
}

\author{
DOUGLAS C. HEGGIE \\ University of Edinburgh, King's Buildings, \\ Edinburgh EH9 3JZ, U.K. \\ PIET HUT \\ Institute for Advanced Study, Princeton, NJ 08540, U.S.A. \\ AND \\ STEPHEN L.W. MCMILLAN \\ Drexel University, Philadelphia, PA 19104, U.S.A.
}

\begin{abstract}
We present results on the exchange cross section for the interaction between a hard binary and a field of single stars, for arbitrary masses. The results are based partly on extensive numerical scattering experiments, and partly on analytic estimates of the mass-dependence of the cross section. They can be used to estimate the rate of exchange in an arbitrary mixture of masses, provided that the binary is hard.
\end{abstract}

\section{Introduction}

In globular clusters, exchange interactions between binary stars and single stars are a plausible mechanism for the formation of low-mass X-ray binaries. The rate of formation depends on the mass function of the stars and binaries present. Relatively little is known about the relevant cross sections for unequal masses. If they are computed by three-body scattering experiments, a fresh set of experiments must be performed for each mass function. Here we summarise a forthcoming paper (Heggie et al. 1995) in which we present approximate cross sections which are valid for arbitrary masses if the binary is hard. Then the rate of formation is given by a simple integration over the mass function. 


\section{Analytical Estimates}

For comparable masses the hard binary exchange cross section is easily estimated by correcting the geometrical area of the binary for gravitational focusing. The result is $\Sigma \sim G M_{123} a / V^{2}$, where $M_{123}$ is the total mass of the three participating stars, $a$ is the initial semi-major axis of the binary, and $V$ is the speed of the intruder relative to the barycentre of the binary, while they are still far apart.

The next step is to investigate how this formula is modified in various extreme mass regimes. For example, suppose the mass of the intruder, $m_{3}$, much exceeds that total mass of the binary, $M_{12}$. Then the binary can be unbound by a tidal interaction, and the corresponding cross section is quite readily estimated. Surprisingly, all these estimates (in the various extreme mass regimes) can be summarised in a single formula, which is given by the coefficient of the exponential in the equation below.

\section{Inclusion of Numerical Data}

We have used a scattering package within the Starlab environment (McMillan \& Hut 1995) to compute accurate cross sections for exchange. Initial conditions were chosen appropriate to hard binaries with a Jeans eccentricity distribution, for a wide variety of masses.

The numerical results have been combined with our analytic estimate in the following semi-numerical fitting formula. Let $x=m_{1} / M_{12}, y=$ $m_{3} / M_{123}$ and $M_{i j}=m_{i}+m_{j}$, where $m_{1}$ is the ejected component. Then

$$
\begin{aligned}
\Sigma & \simeq \frac{G M_{123} a}{V^{2}} \frac{m_{3}^{7 / 2} M_{23}^{1 / 6}}{M_{12}^{1 / 3} M_{13}^{5 / 2} M_{123}^{5 / 6}} \exp \left(3.70+7.49 x-1.89 y-15.49 x^{2}-\right. \\
& \left.-2.93 x y-2.92 y^{2}+3.07 x^{3}+13.15 x^{2} y-5.23 x y^{2}+3.12 y^{3}\right) .
\end{aligned}
$$

This formula fits $75 \%$ of the measurements to better than $20 \%$; larger discrepancies are restricted to mass ratios where the cross sections are probably too small to be of importance in applications. We have also compared our formula with results by other authors, and again the agreement is satisfactory in general. In addition, these comparisons suggest that the above formula is applicable also to circular binaries, except in some parameter ranges where the cross section is very small anyway.

\section{References}

Heggie D.C., Hut P., McMillan S.L.W., 1995, preprint

McMillan S.L.W., Hut P., 1995, preprint 